\title{
Some New Subclasses Of Bi-Univalent Functions Defined By Convolution Associated With Linear Differential Operator
}

\author{
M.Thirucheran ${ }^{1}$, T. Stalin ${ }^{2}$ \\ Associate Professor and Head, Post Graduate and Research Department of Mathematics ${ }^{1}$, Assistant Professor, \\ Department of Mathematics ${ }^{2}$, \\ LN Government College, University of Madras, Chennai, India ${ }^{1}$. \\ Gojan School of Business and Technology, Anna University, Chennai, India ${ }^{2}$. \\ Email:drthirucheran@gmail.com ${ }^{1}$,goldstaleen@gmail.com ${ }^{2}$
}

\begin{abstract}
The main object of this paper is investigating a new subclass of bi-univalent function in the open unit disk U which is defined by convolution of Al-Oboudi Differential Operator. And obtained the initial two Taylor -McLaurin co-efficient $\left|a_{2}\right|$ and $\left|a_{3}\right|$ for the subclass $S_{\Sigma, r}^{m, n, b}$ of Bi-Univalent function.
\end{abstract}

2010 Mathematics Subject classification:30C45,30C55 and 30C80.

Keywords:Analytic functions, univalent functions, bivalent functions, convolution and Al-Oboudi differential operator.

\section{INTRODUCTION AND DEFINITIONS}

\section{Definition 1.1}

Let $A$ denote the class of functions $f$ normalized by

$$
f(z)=z+\sum_{j=2}^{\infty} a_{j} z^{j}
$$

Which are analytic in the open unit disc $\mathrm{U}=\{\mathrm{z} \in C:|z|<1\}$.

For $f \in A$, Al-Oboudi [1] introduces the following operator.

$$
\begin{aligned}
& D^{0} f(z)=f(z), \\
& D^{\prime} f(z)=(1-\delta) f(z)+\delta z f^{\prime}(z)=D_{\delta} f(z), \quad \delta \geq 0 \\
& D^{n} f(z)=D_{\delta}\left(D^{n-1} f(z)\right), \quad n \in N=1,2,3, \ldots . \\
& \therefore D^{n} f(z)=z+\sum_{j=2}^{\infty}[1+(j-1) \delta]^{n} a_{j} z^{j}, \quad n \in N_{0}=N \cup\{0\} . \quad(1.2) \\
& \quad \text { If } \delta=1, \text { then we get Salagean }
\end{aligned}
$$

differential operator.

Koebe One -Quarter Theorem [5]

The range of every function of class $A$ contains the disk of radius $\left\{w:|w|<\frac{1}{4}\right\}$.

It is well known that every function $f \in A$ has an inverse $f^{-1}$ defined by $f^{-1}(f(z))=z$ and $f\left(f^{-1}(w)\right)=w \quad, \quad\left(|w|<r_{0}(f) ; r_{0}(f) \geq \frac{1}{4}\right)$.

For this inverse function $f^{-1}$, we have:

$$
\begin{aligned}
& g(w):=f^{-1}(w)=w-a_{2} w^{2}+\left(2 a_{2}^{2}-a_{3}\right) w^{3} \\
& -\left(5 a_{2}^{3}-5 a_{2} a_{3}+a_{4}\right) w^{4}+\ldots \ldots
\end{aligned}
$$

$$
\text { Let } \quad r(z)=z+\sum_{n=2}^{\infty} r_{j} z^{j} \quad, \quad\left(r_{j}>0\right) \&
$$$$
f(z)=z+\sum_{j=2}^{\infty} a_{j} z^{j} \in S_{\Sigma, r}^{m, n, b, \delta}
$$

Then the Hadamard product $\left(f^{*} r\right)$ defined by if and only if $(f * r)(z)=z+\sum_{j=2}^{\infty} r_{j} a_{j} z^{j} \in S_{\Sigma, r}^{m, n, b, \delta}$

\section{Definition 1.2}

If both the function $f$ and its inverse function $f^{-1}$ are univalent in $U$, then the function $f$ is called bi-univalent.

For example, $\frac{z}{1-z},-1 \circ \mathrm{g}(-z), \frac{1}{2} 1 \circ\left(\mathrm{g} \frac{1+z}{1-z}\right)$, and so on.

However, the familiar Koebe function is not a biunivalent function.

For example, $z-\frac{z^{2}}{2}, \frac{z}{1-z^{2}}$, and so on.

Let the class $\sum$ of bi-univalent function first investigated by Levin [8] and found that Afterward, Brannan and Clunie [2] conjectured that $\left|a_{2}\right| \leq \sqrt{2}$.

Later, Brannan and Taha [3] introduced the new subclass of bi-univalent function of the class $\Sigma$ like the familiar subclasses $S^{*}(\alpha)$ and $C(\alpha)$ of starlike and convex functions of $\alpha .(0 \leq \alpha<1)$ ,respectively. If a function $f \in A$ is in the class $S_{\Sigma}^{*}(\alpha)$ of strongly bi-starlike function of order $\alpha .(0 \leq \alpha<1)$ if each of the following conditions 
satisfied $f(z) \in \sum$ and $\left|\arg \left(\frac{z f^{\prime}(z)}{f(z)}\right)\right|<\frac{\alpha \pi}{2} \quad$, $(z \in U)$ and $\left|\arg \left(\frac{w g(z)}{g(w)}\right)\right|<\frac{\alpha \pi}{2},(w \in U)$

where the function $\mathrm{g}$ is the extension of $f^{-1}$ to $U$ Similarly, a function $f \in A$ is in the class $C_{\Sigma}(\alpha)$ of strongly bi-convex function of order $\alpha$. $(0 \leq \alpha<1)$ if each of the following conditions satisfied $f(z) \in \sum$ and $\left|\arg \left(1+\frac{z f^{\prime \prime}(z)}{f^{\prime}(z)}\right)\right|<\frac{\alpha \pi}{2}$, $(z \in U)$ and $\left|\arg \left(1+\frac{w g^{\prime \prime}(z)}{g^{\prime}(w)}\right)\right|<\frac{\alpha \pi}{2} \quad, \quad(w \in U)$. Where the function $\mathrm{g}$ is the extension of $f^{-1}$ to $U$. For each function $S_{\Sigma}^{*}(\alpha)$ and $C_{\Sigma}(\alpha)$. They found non-sharp estimates on the first two Taylor McLaurin co-efficient $\left|a_{2}\right|$ and $\left|a_{3}\right|$.

Recently, Qing-Hua Xu, Ying-Chun Gui and H.M. Srivastava [9], B.A. Frasin and M.K. Aouf [6], Seker.B [11], and R.M. El-Ashwah [10] investigated some subclasses of bi-univalent function and obtained non-sharp estimates on the first two co-efficient.

By motivated this study we introduce and obtained the initial two co-efficient $\left|a_{2}\right|$ and $\left|a_{3}\right|$ for the subclass $S_{\Sigma, r}^{m, n, b, \delta}$.

\section{Definition: 1.3}

A function $f(z)$ given by (1.1) is said to be in the class $f \in S_{\Sigma, r}^{m, n, b, \delta}(\alpha)$ , $\left(m, n \in N_{0}, m>n, 0<\alpha \leq 1\right)$. if the following conditions are satisfied: $f(z), r(z) \in A$ and $\left|\arg \left(1+\frac{1}{b}\left(\frac{D^{m}(f * r)(z)}{D^{n}\left(f^{*} r\right)(z)}-1\right)\right)\right|<\frac{\alpha \pi}{2}, \quad(z \in U)$

and $\left|\arg \left(1+\frac{1}{b}\left(\frac{D^{m}(g * r)(w)}{D^{n}(g * r)(w)}-1\right)\right)\right|<\frac{\alpha \pi}{2}$,

$(w \in U)$

Where the function $\mathrm{g}$ is given by (1.3).

Definition: 1.4

A function $f(z)$ given by (1.1) is said to be in the class $f \in S_{\Sigma, r}^{m, n, b, \delta}(\gamma)$ , $\left(m, n \in N_{0}, m>n, 0<\alpha \leq 1\right)$, if the following conditions are satisfied: $f(z), r(z) \in A$ and $\mathfrak{R}\left(1+\frac{1}{b}\left(\frac{D^{m}\left(f^{*} r\right)(z)}{D^{n}\left(f^{*} r\right)(z)}-1\right)\right)>\gamma,(z \in U)$
$\& \mathfrak{R}\left(1+\frac{1}{b}\left(\frac{D^{m}(g * r)(w)}{D^{n}(g * r)}-1\right)\right)>\gamma,(w \in U)(1.7)$

Where the function $\mathrm{g}$ is given by (1.3).

\section{Definition: 1.5}

A function $h(z), p(z): U \rightarrow C$ satisfy the conditions $\quad \min \{\mathfrak{R}(h(z)), \mathfrak{R}(p(z))\}>0, \quad z \in U$ and $h(0)=p(0)=1$.

For a function $f \in S_{\Sigma, r}^{m, n, b, \delta}(h(z), p(z))$ defined by (1.1), $\left(m, n \in N_{0}, m>n, 0<\alpha \leq 1\right)$. if the following conditions are satisfied: $f, r \in A$ and $\left(1+\frac{1}{b}\left(\frac{D^{m}(f * r)(z)}{D^{n}(f * r)(z)}-1\right)\right) \in h(z),(z \in U)$

$\&\left(1+\frac{1}{b}\left(\frac{D^{m}(g * r)(w)}{D^{n}(g * r)(w)}-1\right)\right) \in p(z),(w \in U)$

(1.9)

Where the function $\mathrm{g}$ is given by (1.3).

\section{Remarks}

(i). $S_{\Sigma, 1}^{m, n, 1,1}(\alpha)=H_{\Sigma}^{m, n}(\alpha),($ Seker.B [11])

(ii). $S_{\Sigma, 1}^{1,0,1,1}(\alpha)=S_{\Sigma}^{*}(\alpha),($ Brannan and Taha [3])

(iii). $S_{\Sigma, 1}^{2,1,1,1}(\alpha)=C_{\Sigma}(\alpha)$, (Brannan and Taha [3])

\section{MAIN RESULT}

To derive our main results, we should recall the following lemma [4].

\section{Lemma 2.1}

Let $h \in P$ the family of all functions $h$ analytic in $U$ for which $\operatorname{Re}\{h(z)>0\}$ and have the form $h(z)=1+p_{1} z+p_{2} z^{2}+p_{3} z^{3}+\ldots$ for $z \in U$ .Then $\left|p_{n} \leq 2\right|$, for each $n$.

\section{Theorem 2.2}

Let the function $f(z)$ given by (1.1) be in the bi-univalent function class $S_{\Sigma}^{m, n, b, \delta}(h, p)$ .Then

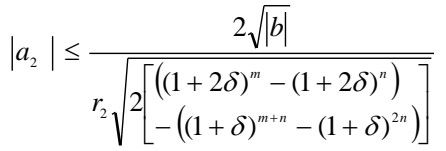

$$
\text { and }\left|a_{3}\right| \leq \frac{1}{r_{3}}\left(\begin{array}{l}
\frac{4|b|^{2}}{\left[(1+\delta)^{m}-(1+\delta)^{n}\right]^{2}} \\
+\frac{2|b|}{\left[(1+2 \delta)^{m}-(1+2 \delta)^{n}\right]}
\end{array}\right)
$$

\section{Proof}

Let consider the function $h$ and $p$ Satisfying the conditions of the definition (1.5) with the form $h(z)=1+h_{1} z+h_{2} z^{2}+h_{3} z^{3}+\ldots \ldots . ., z \in U \&$ $p(w)=1+p_{1} w+p_{2} w^{2}+p_{3} w^{3}+\ldots \ldots . ., w \in U$ respectively. 
International Journal of Research in Advent Technology, Vol.7, No.4, April 2019

E-ISSN: 2321-9637

Available online at www.ijrat.org

Since $f, r \in S_{\sum, r}^{m, n, b, \delta}(h, p)$, then

$\left(1+\frac{1}{b}\left(\frac{D^{m}\left(f^{*} r\right)(z)}{D^{n}\left(f^{*} r\right)(z)}-1\right)\right)=h(z),(z \in U)$

and

$\left(1+\frac{1}{b}\left(\frac{D^{m}(g * r)(w)}{D^{n}(g * r)(w)}-1\right)\right)=p(z),(w \in U)$

respectively.

By equating the coefficient of (2.3 and (2.4), we get

$\left((1+\delta)^{m}-(1+\delta)^{n}\right) a_{2} r_{2}=b h_{1}$

$\left((1+2 \delta)^{m}-(1+2 \delta)^{n}\right) a_{3} r_{3}$

$-\left((1+\delta)^{m+n}-(1+\delta)^{2 n}\right) a_{2}^{2} r_{2}^{2}=b h_{2}$

and

$-\left((1+2 \delta)^{m}-(1+2 \delta)^{n}\right) a_{3} r_{3}$

$+\left[\begin{array}{c}2\left((1+2 \delta)^{m}-(1+2 \delta)^{n}\right) \\ -\left((1+\delta)^{m+n}-(1+\delta)^{2 n}\right)\end{array}\right] a_{2}^{2} r_{2}^{2}=b p_{2}$

From (2.5)\&(2.7),

$$
h_{1}=-p_{1}
$$

And

$2\left[(1+\delta)^{m}-(1+\delta)^{n}\right]^{2} a_{2}^{2} r_{2}^{2}=b^{2}\left(h_{1}^{2}+p_{1}^{2}\right)$

$\therefore a_{2}^{2}=\frac{b^{2}\left(h_{1}^{2}+p_{1}^{2}\right)}{2 r_{2}^{2}\left((1+\delta)^{m}-(1+\delta)^{n}\right)^{2}}$

From $(2.6) \&(2.8)$,

$2\left[\begin{array}{c}\left((1+2 \delta)^{m}-(1+2 \delta)^{n}\right) \\ -\left((1+\delta)^{m+n}-(1+\delta)^{2 n}\right)\end{array}\right] a_{2}^{2} r_{2}^{2}=b\left(h_{2}+p_{2}\right)$

$\therefore \quad a_{2}^{2}=\frac{b\left(h_{2}+p_{2}\right)}{2 r_{2}^{2}\left[\begin{array}{c}\left((1+2 \delta)^{m}-(1+2 \delta)^{n}\right) \\ -\left((1+\delta)^{m+n}-(1+\delta)^{2 n}\right)\end{array}\right]}$

$2\left((1+2 \delta)^{m}-(1+2 \delta)^{n}\right) a_{3} r_{3}$

$=2\left((1+2 \delta)^{m}-(1+2 \delta)^{n}\right) a_{2}^{2} r_{2}^{2}+b\left(h_{2}-p_{2}\right)$

$\therefore a_{3}=\frac{1}{r_{3}}\left(\begin{array}{l}\frac{b^{2}\left(h_{1}^{2}+p_{1}^{2}\right)}{2\left((1+\delta)^{m}-(1+\delta)^{n}\right)^{2}} \\ +\frac{b\left(h_{2}-p_{2}\right)}{2\left((1+2 \delta)^{m}-(1+2 \delta)^{n}\right)}\end{array}\right)$

From Lemma (2.1),(2.11),(2.13) and (2.15), we get

$$
\begin{aligned}
& \left|a_{2}\right| \leq \frac{2 \sqrt{|b|}}{r_{2} \sqrt{2\left[\begin{array}{l}
\left((1+2 \delta)^{m}-(1+2 \delta)^{n}\right) \\
-\left((1+\delta)^{m+n}-(1+\delta)^{2 n}\right)
\end{array}\right]}} \\
& \text { and }\left|a_{3}\right| \leq \frac{1}{r_{3}}\left(\begin{array}{l}
\frac{4|b|^{2}}{\left[(1+\delta)^{m}-(1+\delta)^{n}\right]^{2}} \\
+\frac{2|b|}{\left[(1+2 \delta)^{m}-(1+2 \delta)^{n}\right]}
\end{array}\right)
\end{aligned}
$$

This completes the theorem (2.2).

\section{Theorem 2.3}

Let the function $f, r$ given by (1.1) be in the bi-univalent function class $S_{\Sigma, r}^{m, n, b, \delta}(\alpha)$.Then

$$
\begin{aligned}
& \left|a_{2}\right| \leq \frac{2 \alpha \sqrt{|b|}}{r_{2} \sqrt{2 \alpha\left[\begin{array}{l}
\left((1+2 \delta)^{m}-(1+2 \delta)^{n}\right) \\
-\left((1+\delta)^{m+n}-(1+\delta)^{2 n}\right)
\end{array}\right]}} \\
& \text { and }\left|a_{3}\right| \leq \frac{1}{r_{3}}\left(\begin{array}{l}
\frac{4|b|^{2} \alpha^{2}}{\left[(1+\delta)^{m}-(1+\delta)^{n}\right]^{2}} \\
+\frac{2|b| \alpha}{\left[(1+2 \delta)^{m}-(1+2 \delta)^{n}\right]}
\end{array}\right)
\end{aligned}
$$

(2.17)

\section{Proof}

Let consider the function $h$ and $p$ Satisfying the conditions of the definition (1.5) with the form

$$
h(z)=1+h_{1} z+h_{2} z^{2}+h_{3} z^{3}+\ldots \ldots . ., z \in U
$$

and

$p(w)=1+p_{1} w+p_{2} w^{2}+p_{3} w^{3}+\ldots \ldots ., w \in U$

respectively.

Since $f, r \in S_{\Sigma, r}^{m, n, b, \delta}(\alpha)$, then

$$
\left(1+\frac{1}{b}\left(\frac{D^{m}(f * r)(z)}{D^{n}\left(f^{*} r\right)(z)}-1\right)\right)=[h(z)]^{\alpha},(z \in U)
$$

and $\left(1+\frac{1}{b}\left(\frac{D^{m}(g * r)(w)}{D^{n}(g * r)(w)}-1\right)\right)=[p(z)]^{\alpha},(w \in U)$

respectively.

From Lemma (2.1),(2.11),(2.13) and (2.15), we get

$$
\left|a_{2}\right| \leq \frac{2 \alpha \sqrt{|b|}}{r_{2} \sqrt{2 \alpha\left[\begin{array}{l}
\left.(1+2 \delta)^{m}-(1+2 \delta)^{n}\right) \\
-\left((1+\delta)^{m+n}-(1+\delta)^{2 n}\right)
\end{array}\right]}}
$$

and

$$
\left|a_{3}\right| \leq \frac{1}{r_{3}}\left(\begin{array}{l}
\frac{4|b|^{2} \alpha^{2}}{\left[(1+\delta)^{m}-(1+\delta)^{n}\right]^{2}} \\
+\frac{2|b| \alpha}{\left[(1+2 \delta)^{m}-(1+2 \delta)^{n}\right]}
\end{array}\right)
$$

This completes the theorem (2.3).

\section{Theorem 2.4}

Let the function $f, r$ given by (1.1) be in the biunivalent function class $S_{\Sigma, r}^{m, n, b, \delta}(\gamma)$.Then 


$$
\left|a_{2}\right| \leq \frac{2(1-\gamma) \sqrt{|b|}}{r_{2} \sqrt{2(1-\gamma)\left[\begin{array}{l}
\left((1+2 \delta)^{m}-(1+2 \delta)^{n}\right) \\
-\left((1+\delta)^{m+n}-(1+\delta)^{2 n}\right)
\end{array}\right]}}
$$

$$
\text { and }\left|a_{3}\right| \leq \frac{1}{r_{3}}\left(\begin{array}{l}
\frac{4|b|^{2}(1-\gamma)^{2}}{\left[(1+\delta)^{m}-(1+\delta)^{n}\right]^{2}} \\
+\frac{2|b|(1-\gamma)}{\left[(1+2 \delta)^{m}-(1+2 \delta)^{n}\right]}
\end{array}\right)
$$

Proof

Let consider the function $h$ and $p$ Satisfying the conditions of the definition (1.5) with the form $h(z)=1+h_{1} z+h_{2} z^{2}+h_{3} z^{3}+\ldots \ldots ., z \in U \quad$ and
$p(w)=1+p_{1} w+p_{2} w^{2}+p_{3} w^{3}+\ldots \ldots \ldots, w \in U$ respectively.

Since $f, r \in S_{\Sigma, r}^{m, n, b, \delta}(\gamma)$,then $\left(1+\frac{1}{b}\left(\frac{D^{m}\left(f^{*} r\right)(z)}{D^{n}\left(f^{*} r\right)(z)}-1\right)\right)=\gamma+(1-\gamma) h(z)$, $(z \in U)$ and $\left(1+\frac{1}{b}\left(\frac{D^{m}(g * r)(w)}{D^{n}(g * r)(w)}-1\right)\right)=\gamma+(1-\gamma) p(z)$

$$
\text { , } \quad(w \in U)
$$

respectively.

From Lemma (2.1),(2.11),(2.13) and (2.15), we get

$$
\begin{aligned}
& \left|a_{2}\right| \leq \frac{2(1-\gamma) \sqrt{|b|}}{r_{2} \sqrt{2(1-\gamma)\left[\begin{array}{l}
\left.(1+2 \delta)^{m}-(1+2 \delta)^{n}\right) \\
-\left((1+\delta)^{m+n}-(1+\delta)^{2 n}\right)
\end{array}\right]}} \\
& \text { and }\left|a_{3}\right| \leq \frac{1}{r_{3}}\left(\begin{array}{l}
\frac{4|b|^{2}(1-\gamma)^{2}}{\left[(1+\delta)^{m}-(1+\delta)^{n}\right]^{2}} \\
+\frac{2|b|(1-\gamma)}{\left[(1+2 \delta)^{m}-(1+2 \delta)^{n}\right]}
\end{array}\right)
\end{aligned}
$$

Letting $\delta=1$ in theorem (2.2), (2.3) and (2.4), we obtain the following corollaries.

\section{Corollary 2.5}

Let the function $f(z)$ given by (1.1) be in the bi-univalent function class $S_{\Sigma, 1}^{m, n, b, 1}(h, p)$

Then

$$
\left|a_{2}\right| \leq \sqrt{\left.\frac{2|b|}{\left[\left((3)^{m}-(3)^{n}\right)-\left((2)^{m+n}-(2)^{2 n}\right)\right.}\right]}
$$

$$
\text { and }\left|a_{3}\right| \leq \frac{4|b|^{2}}{\left[(2)^{m}-(2)^{n}\right]^{2}}+\frac{2|b|}{\left[(3)^{m}-(3)^{n}\right]}
$$

\section{Corollary 2.6}

Let the function $f(z)$ given by (1.1) be in the bi-univalent function class $S_{\Sigma, 1}^{m, n, b, 1}(\alpha)$. Then (using definition (1.3))

$\left|a_{2}\right| \leq \sqrt{\left.\frac{2|b| \alpha}{\left[\left((3)^{m}-(3)^{n}\right)-\left((2)^{m+n}-(2)^{2 n}\right)\right.}\right]}$

$$
\text { and }\left|a_{3}\right| \leq \frac{4|b|^{2} \alpha^{2}}{\left[(2)^{m}-(2)^{n}\right]^{2}}+\frac{2|b| \alpha}{\left[(3)^{m}-(3)^{n}\right]}
$$

\section{Corollary 2.7}

Let the function $f(z)$ given by (1.1) be in the bi-univalent function class $S_{\Sigma, 1}^{m, n, b, 1}(\gamma)$. Then (using definition (1.4))

$\left|a_{2}\right| \leq \sqrt{\frac{2|b|(1-\gamma)}{\left[\left((3)^{m}-(3)^{n}\right)-\left((2)^{m+n}-(2)^{2 n}\right)\right]}}$

and $\left|a_{3}\right| \leq \frac{4|b|^{2}(1-\gamma)^{2}}{\left[(2)^{m}-(2)^{n}\right]^{2}}+\frac{2|b|(1-\gamma)}{\left[(3)^{m}-(3)^{n}\right]}$

Letting $\delta=1 \& b=1$ in theorem (2.2), (2.3) and (2.4), we obtain the following corollaries Seker [6].

\section{Corollary 2.8}

Let the function $f(z)$ given by (1.1) be in the bi-univalent function class $S_{\Sigma, 1}^{m, n, 1,1}(h, p)$ .Then $\quad\left|a_{2}\right| \leq \sqrt{\frac{2}{\left.\left[(3)^{m}-(3)^{n}\right)-\left((2)^{m+n}-(2)^{2 n}\right)\right]}}$

$$
\text { and }\left|a_{3}\right| \leq \frac{4}{\left[(2)^{m}-(2)^{n}\right]^{2}}+\frac{2}{\left[(3)^{m}-(3)^{n}\right]}
$$

Corollary 2.9

Let the function $f(z)$ given by (1.1) be in the bi-univalent function class $S_{\Sigma, 1}^{m, n, 1,1}(\alpha)$. Then (using definition (1.3))

$$
\begin{aligned}
& \left|a_{2}\right| \leq \sqrt{\frac{2 \alpha}{\left.\left[(3)^{m}-(3)^{n}\right)-\left((2)^{m+n}-(2)^{2 n}\right)\right]}} \\
& \text { and }\left|a_{3}\right| \leq \frac{4 \alpha^{2}}{\left[(2)^{m}-(2)^{n}\right]^{2}}+\frac{2 \alpha}{\left[(3)^{m}-(3)^{n}\right]}
\end{aligned}
$$

Corollary 2.10

Let the function $f(z)$ given by (1.1) be in the bi-univalent function class $S_{\Sigma, 1}^{m, n, 1,1}(\gamma)$.Then (using definition (1.4))

$$
\begin{aligned}
& \left|a_{2}\right| \leq \sqrt{\frac{2(1-\gamma)}{\left.\left[(3)^{m}-(3)^{n}\right)-\left((2)^{m+n}-(2)^{2 n}\right)\right]}} \\
& \text { and }\left|a_{3}\right| \leq \frac{4(1-\gamma)^{2}}{\left[(2)^{m}-(2)^{n}\right]^{2}}+\frac{2(1-\gamma)}{\left[(3)^{m}-(3)^{n}\right]}
\end{aligned}
$$




\section{ACKNOWLEDGMENT}

The authors thank the referees for their valuable suggestions which lead to the improvement of this paper.

\section{REFERENCES}

[1] Al-Oboudi.(2004), On univalent functions defined by a generalized Salagean operator, Int. Journal of Mathematics and Mathematical Science, Vol.27, 1429--1436.

[2] Brannan D.A, Clunie (1980), Aspects of contemporary complex analysis, Academic press New York, London.

[3] Brannan D.A, Taha T.S (1986), On some classes of bi-valent functions, Studia Univ Babes-Bolyoni Math, Vol.31, 70-77.

[4] Ch. Pommerenke (1975), Univalent functions, Vandenhoeck and Rupercht, Gottingen.

[5] Duren P.L (1983), Univalent functions, Springer-Verlag, New York.

[6] Frasin B.A. and M.K. Aouf (2011), New Subclasses of bi-univalent functions, Applied Mathematics letters, Vol.24, 1569-1573.

[7] G. St. Salagean (1983), Subclasses of univalent functions, Lecture Notes in Math. Vol.1013, Springer-Verlag, Berlin, 362-372.

[8] Lewin M (1967). On a coefficient problem for bi-valent functions, Proc Amer Math Soc, Vol.18, 63-68.

[9] Qing-Hua Xu, Ying-Chun Gui and H.M. Srivastava (2012), Coefficient estimates for a certain subclass of analytic and bi-univalent functions, Applied Mathematics letters, Vol.25, 990-994.

[10]R. M.El-Ashwah, subclass of bi-univalent function defined by convolution, J. Egypt. Math. Soc., 2014, 22, 348-351.

[11] Seker.B (2018), On a new Subclasses of biunivalent functions defined by using Salagean operator, Turk J Math, Vol.42, 2891-2896. 